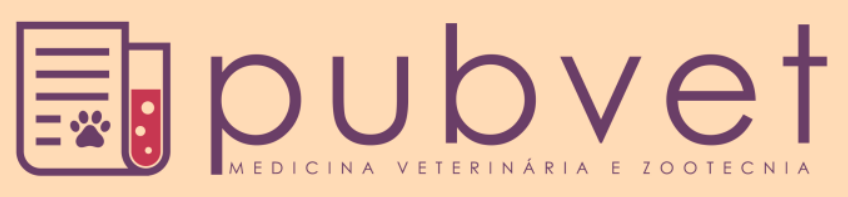

https://doi.org/10.22256/pubvet.v12n1a13.1-5

\title{
Perfil hematológico de caninos recém-nascidos do município de São Luís - Maranhão
}

\author{
Matheus Moreira Ribeiro ${ }^{\bullet}{ }^{1 *}$, Juliana da Silva Alves', Walterlana Sousa Sampaio ${ }^{\bullet 1}$, \\ Ellis de Sousa Barros ${ }^{\bullet}$, Daniel Praseres Chaves ${ }^{\bullet} 2$
}

${ }^{1}$ Acadêmico de Medicina Veterinária, Universidade Estadual do Maranhão (UEMA), Cidade Universitária Paulo VI S/N-Tirirical, São LuísMA 65055-310, Brasil

${ }^{2}$ Professor Adjunto III, Departamento de Patologia, UEMA, Cidade Universitária Paulo VI S/N-Tirirical, São Luís - MA 65055-310, Brasil. E-mail:daniel@cernitas.com.br

*Autor para correspondência: matheus.moreira.ribeiro@hotmail.com

\begin{abstract}
RESUMO. A pediatria como especialidade da clínica médica de pequenos animais vem ganhando espaço à medida que o mercado pet cresce, exigindo novas técnicas de manejo e melhores resultados. Os recém-nascidos representam um desafio especial em terapêutica veterinária, pois diferem marcadamente dos cães adultos. Para que se possa determinar um protocolo terapêutico para um paciente, precisa-se conhecer o estado de saúde do mesmo, sua fisiologia e particularidades. É comum o médico veterinário sentir-se tecnicamente despreparado frente ao recém-nascido, devido esta especialidade não fazer parte da grade curricular na maioria das escolas de medicina veterinária do Brasil. O hemograma é uma importante ferramenta que auxilia o clínico no direcionamento do diagnóstico, pois reflete o estado de saúde do animal no momento da coleta. Permite avaliar se há alteração nos componentes sanguíneos do organismo animal, além de verificar a capacidade de resposta de defesa do organismo frente a alguma ameaça. Foram selecionados para a realização do trabalho animais das raças Rottweiler, Poodle, Labrador e Shi Tzu, sendo 28 machos e 32 fêmeas. O objetivo deste estudo é fornecer subsídios a clínicos veterinários que constantemente em sua rotina de trabalho têm o contato com esta faixa etária, além de contribuir com parâmetros de animais criados no Brasil. Este estudo demonstrou semelhanças entre os resultados obtidos e os valores hematológicos de referência disponíveis na literatura mundial.
\end{abstract}

Palavras chave: Canídeos, hemograma, neonatos

\section{Hematological profile of newborns canines of the municipality of São Luís - Maranhão}

ABSTRACT. The pediatrics as a medical clinical specialty of small animals has been
increasing as the pet market grows, requiring new management techniques and better
results. Newborns are a special challenge in veterinary therapy due to differences they
present when compared to adult dogs. In order to determine a therapeutic protocol for a
patient, it must be known their health condition, physiology and characteristics. Commonly
veterinarians feel technically unprepared towards newborns, because this specialty is not
part of the curriculum in most veterinary schools of our country. A complete blood count
is an important tool that assists in directing clinical diagnosis, since it reflects the animal
health status at the time of collection. It allows assessing whether there are changes in blood
components of the animal body, and checking the organism's defense response to any
threat. Rottweiler, Poodle, Labrador and Shi Tzu breeds were selected for the study, The
objective of this study is to provide grants to veterinarians who constantly in their work 
routine have contact with this age group and also to encourage further research into the consolidation area. This study demonstrated similarities between the results obtained and the hematological reference values available in the world literature.

Key words: canine, complete blood, newborn

\section{Perfil hematológico de recién nacidos caninos del municipio de São Luís - Maranhão}

RESUMEN. La pediatría como especialidad de la clínica médica de pequeños animales viene ganando espacio a medida que el mercado Pet crece, exigiendo nuevas técnicas de manejo y mejores resultados. Los recién nacidos representan un desafío especial en la terapia veterinaria, ya que difieren notablemente de los perros adultos. Para que se pueda determinar un protocolo terapéutico para un paciente, se necesita conocer el estado de salud del mismo, su fisiología y particularidades. Es común que el médico veterinario no se sienta técnicamente preparado frente al recién nacido, debido a que esta especialidad no forma parte de la grade curricular en la mayoría de las escuelas de medicina veterinaria de Brasil. El hemograma es una importante herramienta que auxilia al clínico en la dirección del diagnóstico, pues refleja el estado de salud del animal en el momento de la colecta. Se permite evaluar si hay alteración en los componentes sanguíneos del organismo animal, además de verificar la capacidad de respuesta de defensa del organismo frente a alguna amenaza. Se seleccionaron para la realización del trabajo animales de las razas Rottweiler, Poodle, Labrador y Shi Tzu, siendo 28 machos y 32 hembras. El objetivo de este estudio es proporcionar subsidios a clínicos veterinarios que constantemente en su rutina de trabajo tienen el contacto con este grupo de pacientes, además de incentivar la realización de más investigaciones para la consolidación del área. Este estudio demostró similitudes entre los resultados obtenidos y los valores hematológicos de referencia disponibles en la literatura mundial.

Palabras clave: cachorros, hemograma, neonatos

\section{Introdução}

No âmbito da medicina veterinária, uma das áreas que tem experimentado um crescimento intenso nas últimas décadas é, sem dúvida, a de animais de companhia, os chamados pet's, mais especificamente os cães. A relação entre seres humanos e animais mudou ao longo do tempo e passou a ter grande relevância na sociedade moderna. De cães de guarda e caça, se tornaram verdadeiros companheiros das pessoas, em uma interação de carinho e preocupação. Nos dias atuais, os animais de estimação são considerados como membro da família, já está comprovado após diversos trabalhos os benefícios da interação entre humanos e pets, para ambas as partes, seja para companhia, lazer, participação em terapias ou em políticas de inclusão social. Essa importância dos pets é o principal motivo de desenvolvimento do mercado, que emprega hoje cerca de 1,7 milhão de pessoas (ABINPET, 2015).

A neonatologia é definida como a ciência responsável pelo estudo concernente aos recémnascidos Sorribas (2004). Existe uma definição diversificada para o termo "neonato", diferindo de autor para autor (Davidson, 2003) considerou como neonato, o canino desde o momento do seu nascimento até os dez primeiros dias de idade, para Sorribas (2004) nessa categoria estão os animais até a segunda semana de vida. Já Hoskins (2001) realiza uma abordagem mais genérica ao afirmar que neonato é aquele filhote que depende de sua mãe, para garantir sua sobrevivência, alimentação e outras funções importantes para seu desenvolvimento. Os recém-nascidos diferem dos adultos em vários aspectos e o conhecimento destes é fundamental para formular planos diagnósticos e tratamento. Comumente, o médico veterinário apresenta-se tecnicamente inseguro frente a um recém-nascido doente. Tais pacientes muitas vezes chegam ao veterinário apenas no final do curso de uma doença. A punção venosa pode ser desafiadora e técnicas padrões de exame físico, como auscultação e palpação abdominal são difíceis de serem executadas.

Como nos recém-nascidos caninos não se observa o completo desenvolvimento dos sistemas compensatórios orgânicos, a abordagem 
emergencial deve incluir os cuidados referentes à reversão dos quadros de hipotermia, hipoglicemia e hipovolemia, aumentando dessa forma a sobrevida desses pacientes (Macintire et al., 2005).

A importância da hematologia como meio semiológico, auxiliando os veterinários a estabelecer diagnósticos, firmar prognósticos e acompanhar os tratamentos das inúmeras enfermidades que atingem os animais domésticos é reconhecida e consagrada mundialmente. Entretanto, para que esses objetivos possam ser alcançados e utilizados na plenitude, tornou-se fundamental o conhecimento dos valores de referência do hemograma dos animais sadios, bem como dos fatores causadores de suas variações (Birgel Júnior, 2001). Em cães adultos, as hemácias do sangue periférico, o tecido eritropoético da medula óssea e de sítios extramedulares como o baço e o fígado reage como uma unidade para suprir as necessidades do animal.

O hematócrito do cão recém-nascido aproxima-se do adulto e o número de glóbulos vermelhos, e a concentração de hemoglobina diminuem na medida em que o animal começa a mamar, e continua a decrescer durante o primeiro mês de vida (Hoskins, 2001). A destruição das hemácias é geralmente mais intensa durante as duas primeiras semanas de vida. Devido ao aumento da destruição dos glóbulos brancos e ao rápido crescimento do filhote, o volume total de glóbulos vermelhos circulantes fica reduzido, mas o volume total plasmático permanece relativamente inalterado. Portanto, o animal ao nascer frequentemente se encontra em um estado anêmico devido a sua adaptação ao meio extrauterino. $\mathrm{O}$ número de eritrócitos, a concentração de hemoglobina e o hematócrito continuam a crescer até o animal entrar na fase adulta (Hoskins, 2001).

\section{Material e Métodos}

Participaram do trabalho um total de 60 cães, sendo 28 machos e 32 fêmeas das raças: Rottweiler, Poodle, Labrador e Shi Tzu, distribuídos em três grupos de 20 animais cada, de acordo com a faixa etária destes. Grupo 1: animais de 0 a 15 dias de vida; Grupo 2: de 16 a 30 dias; Grupo 3: de 31 a 45 dias de vida. Antes da realização da coleta de sangue, todos os cães foram avaliados clinicamente, e submetidos a uma inspeção para observar se havia alguma patologia que pudesse alterar ou mascarar os resultados obtidos.

Logo após este procedimento foram colhidas amostras de $5 \mathrm{ml}$ de sangue mediante venopunção jugular, colocando-se $1 \mathrm{~mL}$ em frasco contendo ácido etilenodiamino teratacético (EDTA 10\%) como anticoagulante, para realização do hemograma. Foram confeccionados dois esfregaços em lâminas de microscopia de cada amostra colhida com EDTA para contagem diferencial dos leucócitos e avaliação morfológica das células. Estas lâminas foram secas à temperatura ambiente, identificadas e transportadas ao laboratório de Patologia Clínica Veterinária do CCA/UEMA, onde foram coradas com Rosenfeld modificado e lidas em objetiva de imersão. A contagem global de eritrócitos, o volume globular, a concentração de hemoglobina, os índices hematimétricos, bem como a contagem global de leucócitos e plaquetas foram determinadas utilizando-se sistema hematológico veterinário automático (AH-22-UPSBR). A contagem diferencial de leucócitos foi obtida em esfregaços sanguíneos corados com Rosenfeld modificado.

A avaliação da estrutura de grupos contida nos dados foi executada pela aplicação da estatística multivariada pela análise hierárquica de agrupamento, adotando-se como medida de semelhança a distância euclidiana e como algoritmo de agrupamento o método de Ward (Wilks, 2006). Foi aplicado o teste de tukey para a análise da variância do agrupamento. Os cálculos estatísticos foram realizados com o auxilio do software ASSISTAT 7.7.

\section{Resultados e Discussão}

Após a análise dos resultados obtidos observou-se algumas alterações fisiológicas comuns aos recém-nascidos referentes às hemácias, como a queda no número de eritrócitos na segunda semana de vida do animal. A presença de núcleo, este achado é devido ao crescimento corpóreo acelerado onde os tecidos hematopoiéticos não aumentam a produção sanguínea na mesma proporção (Prats, 2005). A média das hemácias referente aos três grupos analisados (Tabela 1) encontra-se dentro dos valores de referência obtidos por (Meyer, 1991) e não houve variações significativas entre as amostras. Os valores individuais e médios das hemácias sofreram influências significativas do ambiente externo devido as variáveis 
relacionadas: ao clima, pressão atmosférica e a altitude, esse fato é facilmente observado quando os resultados hematológicos de animais de diferentes localidades são comparados.

A média da taxa de hemoglobina por hemácia se encontra dentro dos valores de referência. O volume globular médio (VGM) e a concentração de hemoglobina globular média (CHGM) apresentaram valores reduzidos devido a constante variação fisiológica dos animais desta faixa etária, onde os mecanismos de hematopoiéticos não são tão eficazes até alcançarem dois a três meses de idade. Ao nascimento, o VGM pode variar de 95 a 100fl. O
VGM então diminui, à medida que os glóbulos sanguíneos maiores são eliminados e substituídos por glóbulos vermelhos maduros menores (Hoskins, 2001). De acordo com Meyer (1992) os valores do VGM aos 24 dias já se aproximam dos valores relacionados aos adultos, neste estudo somente próximo aos 30 dias os valores se aproximaram ao parâmetro dos animais adultos concordando com Martins (2005). Em relação ao CHGM, após o nascimento com os pulmões em pleno funcionamento, o sistema hematopoiético precisa se adequar em relação à concentração de hemoglobina na corrente sanguínea, fornecendo hemoglobina suficiente para que as trocas gasosas sejam realizadas de modo eficaz.

Tabela 1. Valores médios e desvio padrão de parâmetros hematológicos de cães recém-nascidos (São Luís - MA)

\begin{tabular}{|c|c|c|c|c|c|c|}
\hline \multirow{2}{*}{ Parâmetros } & \multicolumn{3}{|c|}{ Dias } & \multirow{2}{*}{ VR } & \multirow{2}{*}{ DPM } & \multirow{2}{*}{$\mathrm{CV}, \%$} \\
\hline & $0-15$ & $16-30$ & $31-45$ & & & \\
\hline Hemácias $\left(\mathrm{x} 10^{6} / \mathrm{mm}^{3}\right)$ & $4,71 \pm 0,87 a^{*}$ & $4,36 \pm 0,75 a$ & $4,36 \pm 1,04 a$ & $3,5-6$ & 0,68 & 19,97 \\
\hline Hemoglobina (mg/dL) & $9,25 \pm 1,79 \mathrm{a}$ & $8,73 \pm 1,55 \mathrm{a}$ & $8,63 \pm 2,07 a$ & $8,5-13$ & 1,4 & 20,73 \\
\hline Hematócrito, \% & $30,75 \pm 5,4 \mathrm{a}$ & $28,75 \pm 5,08 \mathrm{a}$ & $28,30 \pm 6,87 \mathrm{a}$ & $26-39$ & 4,59 & 20,63 \\
\hline V.G.M (f/L) & $65,99 \pm 0,17 \mathrm{a}$ & $65,98 \pm 0,16 a$ & $64,92 \pm 0,1 \mathrm{a}$ & $69-83$ & 2,06 & 4,13 \\
\hline C.H.G.M ( \%) & $30,26 \pm 0,68 \mathrm{a}$ & $30,29 \pm 0,41 \mathrm{a}$ & $30,33 \pm 0,35 \mathrm{a}$ & $31-33$ & 0,37 & 1,64 \\
\hline Leucócitos $\left(\mathrm{x} 10^{3} / \mathrm{mm}^{3}\right)$ & $15,7 \pm 7,04 a$ & $15,9 \pm 3,22 \mathrm{a}$ & $12,14 \pm 3,73 a$ & $8-16$ & 3,78 & 34,06 \\
\hline Plaquetas $\left(\times 10^{3} / \mathrm{mm}^{3}\right)$ & $339,76 \pm 134,83 a$ & $309,8 \pm 93,57 \mathrm{a}$ & $393,6 \pm 238,98 \mathrm{a}$ & $200-500$ & 128,79 & 48,66 \\
\hline PPT (g/dL) & $5,82 \pm 0,87 \mathrm{a}$ & $5,59 \pm 0,73 b$ & $6,28 \pm 1,05 a, b$ & $4-6$ & 0,68 & 15,27 \\
\hline Segmentados & $10,9 \pm 2,5 a^{*}$ & $7,8 \pm 3,1 \mathrm{a}$ & $8,6 \pm 1,5 \mathrm{a}$ & $3,8-12,8$ & 2,68 & 9,8 \\
\hline Linfócitos & $2,3 \pm 1,2 \mathrm{a}$ & $4,2 \pm 1,55 \mathrm{a}$ & $4,5 \pm 2,07 \mathrm{a}$ & $2-8$ & 2,4 & 10,33 \\
\hline Monócitos & $1,2 \pm 0,6 \mathrm{a}$ & $0,5 \pm 0,3 \mathrm{a}$ & $0,8 \pm 0,3 \mathrm{a}$ & $0-2$ & 0,59 & 3,45 \\
\hline Eosinófilos & $0,8 \pm 0,17 \mathrm{a}$ & $0,6 \pm 0,16 \mathrm{a}$ & $1,1 \pm 0,1 \mathrm{a}$ & $0-2$ & 0,06 & 4,3 \\
\hline
\end{tabular}

*Letras diferentes na mesma linha indicam diferenças significativas pelo teste de Tukey $(\mathrm{P} \leq 0,05)$. VR: valores de referência; DPM: desvio padrão médio; $\mathrm{CV}, \%$ : coeficientes de variação.

O valor médio dos grupos analisados com relação aos leucócitos permaneceu dentro dos valores de referência, entretanto houve uma variação elevada entre as amostras, que indica a possibilidade de interferência externa que não pode ser controlada como: a ingestão do colostro que tem influência sobre a imunidade do recémnascido $\log$ o nos primeiros dias e a resposta do animal a alguns patógenos com manifestações subclinicas que podem ter passado despercebido pelo processo de triagem. $\mathrm{Na}$ análise dos resultados observou-se uma leucocitose fisiológica onde é caracterizada pela contagem total de leucócitos acima de 20000/1, neutrófilia leve de 15000 a $25000 / 1$ e uma linfocitose de 8000 a 15000/l. Esses dados são provocados pelo estresse do animal no ato da coleta sanguínea, a neutrófilia é atribuída ao aumento do fluxo sanguíneo ocasionado pela excitação do sistema nervoso simpático desencadeado pela epinefrina (Jain, 1986; Hoskins 2001). Na análise da série branca os valores médios dos segmentados, monócitos, linfócitos e eosinófilos permaneceram dentro dos valores de referência, observando-se pequenas elevações apenas na segunda semana de vida.

Os valores com relação a proteína plasmática se encontraram dentro dos valores preconizados pela literatura, entretanto observou-se uma elevação dos valores séricos nas primeiras semanas de vida dos animais, e este fato deve-se a ingestão do colostro que naturalmente fornece ao recém-nascido uma concentração elevada de proteínas. A partir da terceira semana de vida houve uma pequena elevação na média. Nessa idade ocorre $\mathrm{o}$ amadurecimento de órgãos essenciais ao organismo, principalmente o fígado onde ocorre a síntese e a metabolização de 
diversas substâncias, podendo assim aumentar os valores séricos referentes à proteína plasmática.

A anemia pode ser diagnosticada com auxílio de exames complementares como o hemograma, observando o número de eritrócitos, a taxa de hemoglobina e a porcentagem do hematócrito, associado ao exame físico do paciente. Os valores médios dos três parâmetros citados se mantiveram dentro dos valores de referência da literatura. Entretanto nota-se que uma determinada quantidade de animais de cada grupo apresentou os parâmetros de hemácias, hematócrito e hemoglobina abaixo dos valores de referência para a idade (Figura 1), além de apresentarem estados nutricionais e físicos ótimos, caracterizando assim um quadro de anemia fisiológica. Observa-se que o grupo 3 apresentou a maior porcentagem de animais com os três parâmetros abaixo dos valores de referência, e esta variação pode estar relacionada com a grande instabilidade fisiológica deste período onde ocorre o crescimento do animal e o amadurecimento da maioria dos sistemas orgânicos. Segundo Martins (2005) a anemia fisiológica pode estar relacionada também a deficiência nutricional, ocasionada pela baixa quantidade de ferro presente no leite materno e pela hemodiluição causada pelo aleitamento, esta instabilidade pode ser refletida no hemograma ocasionando variações entre as amostras.

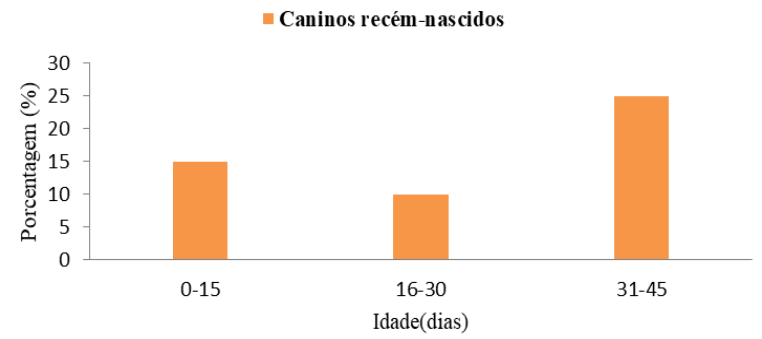

Figura 1. Porcentagem de animais por grupo $n=20$ que apresentaram os parâmetros de hemácias, hemoglobina e hematócrito abaixo dos valores de referência.

\section{Conclusão}

Este estudo demonstrou semelhanças entre os resultados obtidos e os valores hematológicos de referência disponíveis na literatura mundial, indicando mínima influência regional, racial e ambiental sobre os dados hematológicos de cães recém-nascidos.

\section{Referências Bibliográficas}

ABINPET - Associação Brasileira da Indústria de produtos para Animais de Estimação. 2015. Mercado. São Paulo: Stilo.
Birgel Júnior, E. H., Dangelino, J. L., Benesi, F. J. 2001. Valores de referência do eritrograma de bovinos da raça Jersey criados no Estado de São Paulo. Arquivo Brasileiro de Medicina Veterinária e Zootecnia, 53, 164-171.

Davidson, A. P. 2003. Approaches to reducing neonatal mortality in dogs In: (Ed.). Recent advances in small animal reproduction. Ithaca, NY: International Veterinary Information Service.

Hoskins, J. D. 2001. Puppy and kitten losses.In: Hoskins JD (Ed.). Veterinary pediatrics dogs and cats from birth to six months. 3.ed. Philadelphia: WB Saunders, 57-61.

Jain, N. C. 1986. Veterinary Hematology. 4 (Ed.).California: Lea \&Febiger, 765p.

Macintire, D. K., Drobatz, K. J., Haskins, S. C., Saxon, W. D. 2005. Manual of Small Animal Emergency and Critical Care Medicine, Baltimore: Lippincott Williams e Wilkins, p.341-352.

Martins, R. R. 2005. Hemograma, proteinograma e enzima gamaglutamiltransferase em cães neonatos, do $3^{\circ}$ ao $45^{\circ}$ dia de vida, sob a ação da idade e da suplementação com luteína. 71f. Dissertação, Universidade Estadual Paulista, Botucatu.

Meyer, J. D., Coles, E. H. \& Rich, L. J. 1992. Veterinary Laboratory Medicine: interpretation and Diagnosis. Philadelphia: W.B. Saunders, 350p.

Prats A. 2005. Farmacologia e terapêutica veterinária. In: Prats A. (Ed.). Neonatologia e pediatria canina e felina. Mad.

Sorribas, C. E. 2004. Neonatología canina. In: Gobello, C. (Ed.). Temas de reproducción de caninos y felinos por autores latinoamericanos. 2. ed. Buenos Aires: Gráfica Latina, p.151-163.

Wilks, D. S. 2006. Statistical Methods in the G. B. Simulação de séries de dados Atmospheric Sciences. International Geophysics climatológicos usando análise de componentes Series, Vol. 91, Elsevier.

Article History:

Received 9 September 2017

Accepted 9 October 2017

Available online 24 November 2017

License information: This is an open-access article distributed under the terms of the Creative Commons Attribution License 4.0, which permits unrestricted use, distribution, and reproduction in any medium, provided the original work is properly cited. 